\title{
Expériences diverses en francophonie
}

Nicole Rege Colet et Christelle Lison

\section{OpenEdition}

Journals

Édition électronique

URL : http://journals.openedition.org/ripes/983

DOI : 10.4000/ripes.983

ISSN : 2076-8427

\section{Éditeur}

Association internationale de pédagogie universitaire

\section{Référence électronique}

Nicole Rege Colet et Christelle Lison, «Expériences diverses en francophonie », Revue internationale de pédagogie de l'enseignement supérieur [En ligne], 31(2) | 2015, mis en ligne le 01 juillet 2015, consulté le 23 septembre 2020. URL : http://journals.openedition.org/ripes/983 ; DOI : https://doi.org/10.4000/ ripes. 983

Ce document a été généré automatiquement le 23 septembre 2020.

Article L.111-1 du Code de la propriété intellectuelle. 


\title{
Expériences diverses en francophonie
}

\author{
Nicole Rege Colet et Christelle Lison
}

1 Les cinq articles de ce deuxième numéro 2015 de RIPES illustrent la diversité des thèmes qui animent la communauté de l'Association internationale de pédagogie universitaire (AIPU) et les réseaux de travail qui unissent la Belgique, la France, le Québec et la Suisse. Or, il convient de se rappeler que la communauté francophone ne se limite pas à ces quatre pays et que l'AIPU a toujours eu pour vocation de tisser des liens solides au sein de toutes les régions francophones. Dès lors, où sont les auteurs du continent africain? Que deviennent les propositions émanant des sections de l'Algérie, du Liban, du Maroc ou de la Tunisie?

2 Force est de reconnaître que si nous recevons de nombreuses propositions de ces pays et, en particulier, de la part de doctorants désireux de publier afin de pouvoir soutenir leur thèse, la qualité des textes soumis est loin de répondre aux exigences d'une revue scientifique avec comité de lecture. Quand c'est possible, nous proposons un accompagnement pour guider les auteurs novices dans les méandres de la publication scientifique de haut niveau. Le travail pour arriver à un texte abouti peut alors prendre un certain temps. N'en déduisez pas pour autant qu'il y a une fracture Nord-Sud dans la production de textes scientifique dans le domaine de la pédagogie de l'enseignement supérieur! Les difficultés rencontrées peuvent tout aussi bien se manifester en France, au Maroc, en Suisse et au Sénégal.

3 Nous défendons notre ligne de travail qui consiste à publier des textes scientifiques de qualité et, par moments, nous pouvons être très directives dans la phase d'édition. Notre intention est de maintenir les critères qualité et de ne pas faire une évaluation de complaisance. Notre but? Faire en sorte qu'une publication dans RIPES contribue à la valorisation des travaux des auteurs et à l'avancement dans la carrière. Nous invitons le lecteur à prendre connaissance de ce numéro composé d'articles rédigés par des personnes moins expérimentées ayant bénéficié de l'accompagnement de personnes plus expérimentées. Ils témoignent de ce qu'il est possible de faire lorsque l'on s'ouvre à la collaboration entre pairs pour s'aider à grandir. Augurons que le prochain colloque 
de l'AIPU, qui se tiendra dans moins d'une année en Tunisie, soit le départ de nouveaux projets de publications conjointes au sein de la communauté francophone.

Bernard Coulibaly et Hélène Hermann de l'Université de Haute Alsace en France s'intéressent aux attentes des étudiants et des enseignants vis-à-vis du prochain Learning Center qui sera mise en place dans leur université. Partant du principe que les usagers adopteront et investiront un espace de travail innovant si ce dernier répond à leurs besoins, ils sont allés à l'écoute des attentes et des besoins les plus importants. Les résultats de leur enquête montrent que les étudiants et les enseignants-chercheurs sont à la recherche de choses différentes. Si les premiers valorisent les dimensions en lien avec la socialisation (lieu de vie, convivialité et sentiment d'appartenance), les enseignants-chercheurs le considèrent comme un outil indispensable pour leur activité de recherche. Reste alors à faire de ce lieu en émergence un espace de rencontres entre enseignement et apprentissage pour que le mot Learning (apprentissage) ne soit pas superflu.

5 Le deuxième article résulte de la réflexion commune menée entre les conseillers pédagogiques de deux institutions, l'une belge et l'autre suisse, sur le cadre d'accompagnement mis en place dans chacune des institutions pour soutenir les enseignants dans le développement de la recherche appliquée sur leurs enseignements et leurs pratiques pédagogiques. Le dialogue entre l'Ecole Libre Mosane en Belgique, porté par Sandrine Biémar, Déborah Malengrez et Laurence Oger, et l'Université de Lausanne en Suisse, porté par Amaury Daele, permet de cerner la notion de Scholarship of Teaching and Learning (SoTL) telle qu'elle se manifeste dans leurs établissements tout en soulignant l'attention que les enseignants portent à la recherche sur leurs pratiques pédagogiques pour leur développement professionnel. Les auteurs illustrent comment les mesures d'accompagnement prévus dans leurs institutions leur permettent de consolider leur rôle de conseiller pédagogique auprès des enseignants.

6 Julien Morand, France Landry, Georgette Goupil et Nicole Bonenfant, tous les quatre de l'Université du Québec à Montréal, nous présentent les résultats d'une recherche sur les perceptions d'étudiants tuteurs recrutés pour accompagner des étudiants avec des handicaps dits non visibles tels que les troubles de l'apprentissage, les troubles de l'attention, les troubles du spectre de l'autisme et d'autres troubles de la santé mentale. Les résultats indiquent que les étudiants tuteurs souhaiteraient bénéficier d'une supervision pour rester dans le cadre de leur accompagnement pédagogique et éviter de glisser vers une relation d'aide à autrui.

7 L'article de Viviane Vierset, Mariane Frenay et Denis Bédard, deux auteurs belges et un auteur québécois, s'intéresse aux critères à utiliser pour questionner la qualité pédagogique des stages cliniques en formation médicale de l'Université de Liège. Pour ce faire, ils utilisent le cadre conceptuel de l'apprentissage et de l'enseignement contextualisé authentique en illustrant les deux principes fondamentaux de celui-ci, soit l'authenticité du contexte d'apprentissage et le compagnonnage cognitif, à travers le cas de la gynécologie-obstétrique. Ce travail réflexif les amène à soulever trois défis pour la formation: (1) réfléchir sur les postures pédagogiques afin de diminuer la distance « socio-pédagogique » entre un superviseur qui sait et un stagiaire qui ne sait pas, (2) amener le stagiaire à prendre une posture plus autonome et réflexive dans le contexte professionnel, et (3) développer la formation des superviseurs au niveau institutionnel afin que ceux-ci questionnent leurs pratiques pédagogiques. 
8 Le dernier article est celui de Martine Pons-Desoutter, aujourd'hui de l'Université Grenoble 3. Dans son parcours professionnel, elle a eu l'occasion de travailler au sein de l'Université de la Nouvelle-Calédonie. Comme nombre d'universités françaises, celle-ci tente de remédier à l'échec massif des étudiants de première année de licence. C'est dans ce cadre qu'une enquête a été menée, à l'aide entretiens, auprès des étudiants et des enseignants afin de dégagés des axes d'amélioration. Parmi les pistes à suivre : la mobilisation des pédagogies actives, l'intégration d'outils multimédias dans les cours, le développement de l'auto-évaluation, des approches par projets et par compétences, une clarification du contrat didactique, l'usage de l'évaluation des enseignements pour faire évoluer les contenus de formation.

9 Strasbourg et Sherbrooke, le 10 juillet 2015

Nicole Rege Colet et Christelle Lison

\section{AUTEURS}

\section{NICOLE REGE COLET}

Strasbourg

CHRISTELLE LISON

Sherbrooke 